BULLETIN (New Series) OF THE

AMERICAN MATHEMATICAL SOCIETY

Volume 45, Number 2, April 2008, Pages 285-291

S 0273-0979(08)01196-8

Article electronically published on January 22, 2008

\title{
TAIT'S CONJECTURES AND ODD CROSSING NUMBER AMPHICHEIRAL KNOTS
}

\author{
A. STOIMENOW
}

\begin{abstract}
We give a brief historical overview of the Tait conjectures, made 120 years ago in the course of his pioneering work in tabulating the simplest knots, and solved a century later using the Jones polynomial. We announce the solution, again based on a substantial study of the Jones polynomial, of one (possibly his last remaining) problem of Tait, with the construction of amphicheiral knots of almost all odd crossing numbers. An application to the non-triviality problem for the Jones polynomial is also outlined.
\end{abstract}

\section{The FiRst KNOT TABLES}

Knot theory originated in the late 19th century. At that time, W. Thomson ("Lord Kelvin"), P. G. Tait and J. Maxwell propagated the vortex-atom theory in an attempt to explain the structure of the universe. They believed that a supersubstance, ether, makes up all of matter, and atoms are knotted tubes of ether. Knotting is hereby understood as tying a piece of rope and then identifying both its ends so that the tying cannot be any more undone.

Thus, in the realm of constructing a periodic table of elements, Tait began the catalogization of the simplest knots. He depicted knots (as we still do today) by diagrams consisting of a (smooth) plane curve with transverse self-intersections, or crossings. At each crossing one of the two strands passes over the other. The term "simplest" refers to the number of crossings of the diagram. We can define the crossing number of a knot as the minimal crossing number of all its diagrams, and say that Tait sought the list of knots with given (small) crossing number. The list meant to present each knot by exactly one diagram. This entails that knots from different diagrams should be inequivalent, in the sense that one cannot turn a (closed) piece of rope knotted one way into one knotted the other way without cutting the rope. The simplest knots are shown in figure 1. The leftmost one, of crossing number 0 , is the trivial knot or unknot. It has some special importance, much like the unit element in a group.

Tait completed the list up to 7 crossings. Little, Kirkman, later Conway Co and others took over and continued his work. In the modern computer age, tables have reached the knots of 17 crossings, with millions of entries, even though Tait's vortex-atom theory has long been dismissed. An account on knot tabulation is

Received by the editors May 30, 2007.

2000 Mathematics Subject Classification. Primary 57M25; Secondary 01A55, 01A60.

Key words and phrases. Jones polynomial, amphicheiral knot, crossing number.

Financial support by the 21st Century COE Program is acknowledged.

(C)2008 American Mathematical Society Reverts to public domain 28 years from publication 


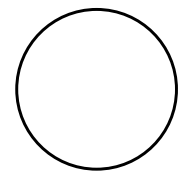

unknot

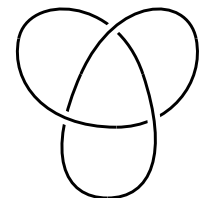

left-hand trefoil

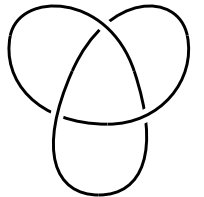

right-hand trefoil

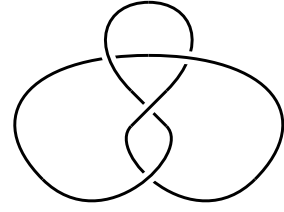

figure-8 knot

Figure 1

given, with emphasis on its history, in $[\mathbf{S}$, and from a more contemporary point of view in $\mathrm{HTW}, \mathrm{H}$.

\section{TAIT'S CONJECTURES}

Tait's accomplishment allows us to call him with some justification the first knot theorist. Yet Tait worked mainly by intuition. He had at his time no rigorous way of showing knots inequivalent. Tait's reasoning is not easy to interpret precisely nowadays, since it had been formulated in a language quite different from (and far less developed than) our present usage. Nonetheless he evidently observed several phenomena, which, apart from knot tabulation, would become a legacy to his successors.

It remains unclear whether Tait was convinced certain properties hold for all or just for alternating knots. A knot is alternating if in some (alternating) diagram the curve passes crossings interchangingly over-under like $-\mid \frac{1}{\mid}$, i.e. not containing $-|-|-$, or $\frac{1}{1} \frac{1}{1}$. The knots in figure 1 are such. In fact, this is true for all knots up to 7 crossings, catalogued by Tait, and at least for a large portion of the slightly more complicated ones he was shown by his successors in his lifetime (even though it is known now that alternation is a rare property for generic crossing numbers [Th3]). Thus, certainly Tait was guided by evidence from alternating diagrams. Their occurrence in the tables suggested to him

Conjecture 2.1 (Tait's conjecture I). A reduced, i.e. not of the form alternating diagram has minimal crossing number (for the knot it represents).

For the next problem, we need to define the writhe. If one equips (the curve of) a knot diagram with an orientation, then each crossing looks, if observed from an appropriate angle, locally like $\searrow$ ( positive crossing) or $\searrow$ (negative crossing). The writhe is the difference between the number of the former crossings and the number of the latter. (An easy observation shows that the writhe is the same for either orientation.)

Conjecture 2.2 (Tait's conjecture II). Minimal crossing number diagrams of the same (alternating?) knot have the same writhe.

For alternating diagrams, he conjectured more precisely the following: 
Conjecture 2.3 (Tait's conjecture III). Alternating diagrams of the same knot are related by a sequence of flypes:

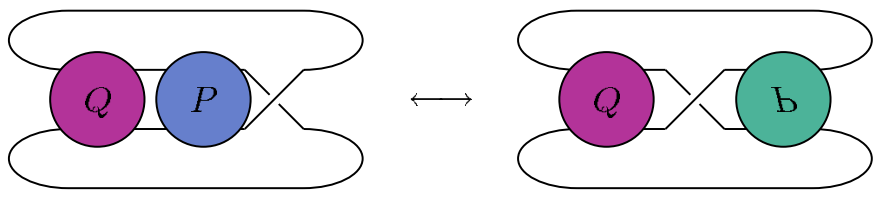

One can easily observe that a flype preserves the writhe, and so conjecture III implies conjecture II (for alternating knots) 1

For Tait's last problem, we consider an amphicheiral knot. Such a knot can be turned into its mirror image. From the knots in figure 1 the unknot is obviously amphicheiral. So is the figure-eight knot, as can be shown by a simple exercise. In contrast, the trefoil is not amphicheiral. In other words, the left-hand trefoil and its mirror image, the right-hand trefoil, are two distinct knots (a fact that stubbed knot theorists for a while and was first proved by Max Dehn). Tait was wondering what crossing numbers amphicheiral knots can have. The evidence he had in mind can probably be formulated so:

Conjecture 2.4 (Tait's conjecture IV). Amphicheiral (alternating?) knots have even crossing number.

Note that (for alternating knots and with the remark in footnote 1) this is a consequence of conjecture II, for mirroring a diagram interchanges positive and negative crossings, and so negates the writhe.

\section{REIDEMEISTER MOVES AND INVARIANTS}

A few decades after their genesis, Tait's knot lists would be proved right. With the work of Alexander, Reidemeister and others, knot theory began to be put on a mathematical fundament. Reidemeister showed that three types of local moves (i.e. moves altering only a fragment of the diagrams, as shown below) suffice to interrelate all diagrams of the same knot.

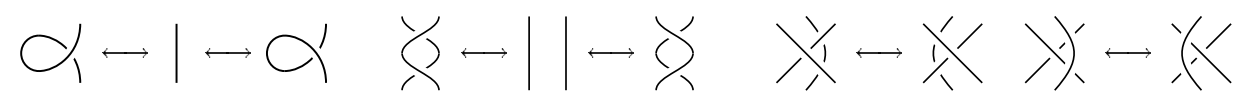

To distinguish two knots thus translates into the question how to prove that two diagrams (of these knots) are not connected by a sequence of such moves. This is done with the help of an invariant, that is, a map

$\{$ knot diagrams $\} \rightarrow$ some domain

whose value in the domain does not change (is invariant) when the argument (diagram) is changed by a Reidemeister move. It is justified to ask what the value domain should be. The answer is that it would suffice to be any set of objects whose distinctness is easy to verify, yet which is large enough to allow the invariant to take many different values. For us it will be the ring $\mathbb{Z}\left[t, t^{-1}\right]$ of Laurent polynomials in one variable with integer coefficients. Clearly one can compare coefficients easier than wondering about a sequence of Reidemeister moves, which may be arbitrarily long and pass over arbitrarily complicated intermediate diagrams. (There is a

\footnotetext{
${ }^{1} \mathrm{Up}$ to some technical issues of primeness and whether all minimal crossing diagrams are alternating. These issues are settled, but we like to skip them here for simplicity.
} 
fundamental, but out of our focus here, issue that one can to some extent control these sequences, and how to do so $[\mathrm{HL}$.) Another obvious desirable feature of an invariant is that we could easily evaluate it from a diagram.

Alexander's merit was to construct precisely such an invariant. The Alexander polynomial [Al] remained (and still remains) a main theme in knot theory for decades to come. Let us observe, though, that we actually already came across one other knot invariant (here with values in $\mathbb{N}$ ), the crossing number. It is an invariant directly by definition, since it was defined on the whole Reidemeister move equivalence class of diagrams. However, this definition makes it difficult to evaluate from a diagram, in contrast to Alexander's polynomial, for which its creator, and later many others, gave several simple procedures.

\section{The Jones POLYNOMial}

Sixty years after Alexander, a new chapter of knot theory was opened by V. Jones, with the discovery of a successor to Alexander's polynomial. The developments the Jones polynomial $V[\mathrm{~J}]$ has sparked in the 20 years since its appearance (Vassiliev and quantum invariants, etc.) are impossible to be even vaguely sketched in com-

pleteness, and go far beyond both the competence and expository intention of the author here. Several more concepts (braids, links, geometric features) are left out for simplicity and length reasons, for which the author would like to apologize at this place. A good related (though still partial and now no longer very recent) account was given by Birman $\mathrm{Bi}$.

Let us recall, though, one of the first achievements the Jones polynomial became famous for - the solution of Tait's conjectures for alternating knots. Conjectures I, II and IV were proved by Kauffman Kf2, Murasugi $\mathrm{Mu}$, $\mathrm{Mu} 2$ and Thistlethwaite Th2]. We will need a few more words on Kauffman's proof, since it uses a calculation procedure for $V$ called state model. This state model also gives a very elementary proof that the Jones polynomial is an invariant. (Kauffman had previously developed a similar model for the Alexander polynomial, too Kf3.)

Based on Kauffman's state model, Lickorish and Thistlethwaite [LT] defined a (semi)adequate knot and diagram. An advantage of this concept is that alternating diagrams/knots are adequate. Many details would better be skipped here, but let us say that a diagram is adequate/semiadequate if it is +adequate and/or -adequate, and that taking the mirror image of a diagram transforms the property for + into the one for -. A knot with some of these properties is defined as one that has a diagram with the feature of the same name. Thistlethwaite extended the proof of Tait's conjectures I, II and IV to the class of adequate knots Th, applying a 2-variable generalization of $V$, the Kauffman polynomial [Kf.

For a general (non-alternating) knot, in the case of conjecture II, Tait's intuition has been proved misleading. In the 1970's, K. Perko [Pe observed that there are two 10 crossing knots in the tables [Ro, appendix], which are equivalent, even though their 10 crossing diagrams have different writhe. This duplication had remained unnoticed for quite a while, possibly due to the belief in Tait's conjecture. Remedying this error (and a few other long-remaining ones) in the tables still causes some confusion in their use.

Tait's conjecture III was settled a few years after the others by Menasco and Thistlethwaite [MT], mostly using geometric techniques, though again with some (now subordinate) appearance of the Jones polynomial. 


\section{THE CROSSING NUMBERS OF AMPHICHEIRAL KNOTS}

Kauffman, Murasugi and Thistlethwaite's proof of Tait's conjecture IV shows that an alternating knot $K$ of odd crossing number and its mirror image $! K$ always have distinct Jones polynomials. (Let us in contrast remark that $K$ and $! K$ have the same Alexander polynomial for every knot $K$.) In the opposite direction, their work allows one to easily find a(n alternating) amphicheiral knot of every even crossing number at least 4 . But their results could not decide what crossing number nonalternating amphicheiral knots can have. The main aim of this note is to announce the complete solution to Tait's (presumably last) problem.

Theorem 5.1. For each odd natural number $n \geq 15$, there exists an amphicheiral knot of crossing number $n$.

Similarly to Perko's knot, a particular instance disproving Tait's conjecture IV for non-alternating knots was found accidentally: Hoste and Thistlethwaite, in the course of routine knot tabulation, discovered an amphicheiral 15 crossing knot. (Their compilational work had previously shown that there are no amphicheiral knots of odd crossing numbers up to 13.) Settling the other crossing numbers is a major problem, though, since exhaustive enumeration is no longer a feasible approach - we face the above-noticed difficulty that we do not know (generally) how to determine the crossing number. A few other methods are known, but all fail on such examples. Thus the way to our result is rather far, and below we will conclude by just giving a brief outline of the proof. The details will appear in a separate (long) paper. We also mention another application of our approach, which addresses the non-triviality problem for the Jones polynomial.

\section{Semiadequacy invariants AND the NON-TRIVIALity PROBlem}

The Alexander polynomial was, from its very beginning, connected to topological features of knots. The situation is rather different for its successor. The problem of giving a topological meaning to the Jones polynomial has bothered many knot theorists ever since this invariant appeared. So far we still find ourselves in the embarrassing state where we "can quickly fill pages with the coefficients and exponents of $V$ for not-too-complicated knots without having the slightest idea what they mean" ([Bi, end of $\S 3])$. Similarly open, and intriguing, remains the question of Jones whether his polynomial detects the unknot. Again, "our lack of knowledge about this problem is in striking contrast to the control mathematicians now have over the Alexander polynomial: understanding its topological meaning, we also know precisely how to construct knots with Alexander polynomial 1" (ibid., rem. (iii) after theorem $3, \S 8$; see, though, also [EKT]).

In an attempt to gain more insight into the appearance of the Jones polynomial, the author [St], and (up to minor interaction, independently) Dasbach and Lin DL, DL2, initiated a detailed study of some coefficients of $V$ in semiadequate

diagrams. Let us remark here that, while adequacy is only a slight extension of alternation, semiadequacy is a rather wide extension of adequacy. (For the experts: semiadequate knots contain completely positive, Montesinos and 3-braid knots.) Semiadequacy is still a fairly general condition, yet it helps settle many technical issues.

For semiadequate knots the first coefficient of the Jones polynomial is \pm 1 , almost by definition $[\mathrm{LT}]$. The outcome of our work was that we gained an understanding 
of coefficients 2 and 3 . Their invariance allows us to derive 3 invariant quantities each from a +adequate, and similarly from a -adequate diagram, called below semiadequacy invariants. Their merit is that they reflect directly certain features of the diagram, and so we have a precise idea what a semiadequate diagram with given invariants must look like. The first invariant allows us to prove:

Theorem 6.1. Semiadequate knots have non-trivial Jones polynomial.

This implies (for experts) the result also for Montesinos and 3-braid knots, but it can be proved also for their Whitehead doubles, some strongly $n$-trivial knots and $k$-almost positive knots with $k \leq 3$.

Our three semiadequacy invariants become also, joined by a relative obtained from the Kauffman polynomial and Thistlethwaite's results Th, the main tool for the proof of Theorem 5.1. For given odd $n \geq 15$, we start with an amphicheiral knot $K$ that has an $n$ crossing diagram, which is semiadequate. Luckily, such examples can be obtained by leaning on Hoste-Thistlethwaite's knot. The work in Th shows then that the crossing number of $K$ is at least $n-1$, and were it $n-1$, a minimal crossing diagram $D$ would be adequate. Then we have 4 invariants for both + adequacy and -adequacy each available. A detailed study of what an $n-1$ crossing diagram with such invariants must look like is necessary to exclude most cases for $D$. Hereby, among the various generalizations of Thistlethwaite's knot, one must choose carefully the one whose invariants make the exclusion argument most convenient (or better to say, feasible at all). Only a small fraction of possibilities for $D$ remain, which are easy to check and rule out by computer. This allows us to conclude that in fact $D$ cannot exist.

\section{About the AUTHOR}

Alexander Stoimenow graduated from Freie University Berlin, Germany, and is a researcher at the Research Institute for Mathematical Sciences in Kyoto, Japan. He is currently a part-time researcher at the Advanced Mathematical Institute, Osaka City University, Japan.

\section{REFERENCES}

[Al] J. W. Alexander, Topological invariants of knots and links, Trans. Amer. Math. Soc. 30 (1928), 275-306. MR.1501429

[Bi] J. S. Birman, New points of view in knot theory, Bull. Amer. Math. Soc. 28(2) (1993), 253-287. MR.1191478 (94b:57007)

[Co] J. H. Conway, On enumeration of knots and links, in "Computational Problems in Abstract Algebra" (J. Leech, ed.), Pergamon Press, 1969, 329-358. MR0258014 (41:2661)

[DL] O. Dasbach and X.-S. Lin, A volume-ish theorem for the Jones polynomial of alternating knots, math.GT/0403448, to appear in Pacific J. Math.

[DL2] On the Head and the Tail of the Colored Jones Polynomial, Compositio Math. 142(5) (2006), 1332-1342. MR2264669 (2007g:57018)

[EKT] S. Eliahou, L. H. Kauffman and M. Thistlethwaite, Infinite families of links with trivial Jones polynomial, Topology 42(1) (2003), 155-169. MR1928648(2003g:57015)

[HL] J. Hass and J. C. Lagarias, The number of Reidemeister moves needed for unknotting, J. Am. Math. Soc. 14(2) (2001), 399-428. MR.1815217 (2001m:57012)

$[\mathrm{H}] \quad$ J. Hoste, The enumeration and classification of knots and links, "Handbook of Knot Theory" (W. Menasco and M. Thistlethwaite, eds.), Elsevier (2005), 209-232. MR2179263 (2006k:57012)

[HTW] J. Hoste, M. Thistlethwaite and J. Weeks, The first 1,701,936 knots, Math. Intell. 20(4) (1998), 33-48. MR 1646740(99i:57015) 
[J] V. F. R. Jones, A polynomial invariant of knots and links via von Neumann algebras, Bull. Amer. Math. Soc. 12 (1985), 103-111. MR766964 (86e:57006)

[Kf] L. H. Kauffman, An invariant of regular isotopy, Trans. Amer. Math. Soc. 318 (1990), 417-471. MR958895 (90g:57007)

[Kf2] _ State models and the Jones polynomial, Topology 26 (1987), 395-407. MR899057 (88f:57006)

[Kf3] , Formal knot theory, Mathematical Notes 30, Princeton University Press, Princeton, NJ, 1983. MR712133 (85b:57006)

[LT] W. B. R. Lickorish and M. B. Thistlethwaite, Some links with non-trivial polynomials and their crossing numbers, Comment. Math. Helv. 63 (1988), 527-539. MR.966948 (90a:57010)

[MT] W. W. Menasco and M. B. Thistlethwaite, The Tait flyping conjecture, Bull. Amer. Math. Soc. 25 (2) (1991), 403-412. MR1098346 (92b:57017)

[Mu] K. Murasugi, Jones polynomial and classical conjectures in knot theory, Topology 26 (1987), 187-194. MR895570 (88m:57010)

[Mu2] _ Jones polynomials and classical conjectures in knot theory II, Math. Proc. Cambridge Philos. Soc. 102(2) (1987), 317-318. MR898151 (88m:57011)

[Pe] K. A. Perko Jr., On the classification of knots, Proc. Amer. Math. Soc. 45 (1974), 262266. MR0353294 (50:5778)

[Ro] D. Rolfsen, Knots and links, Publish or Perish, 1976. MR0515288 (58:24236)

[S] D. S. Silver, Knot Theory's Odd Origins, American Scientist 94(2) (2006), 158-165.

[St] A. Stoimenow, On polynomials and surfaces of variously positive links, Jour. Europ. Math. Soc. 7(4) (2005), 477-509. MR2159224 (2006d:57014)

[Th] M. B. Thistlethwaite, On the Kauffman polynomial of an adequate link, Invent. Math. 93(2) (1988), 285-296. MR948102 (89g:57009)

[Th2] _ A spanning tree expansion of the Jones polynomial, Topology 26(3) (1987), 297-309. MR899051 (88h:57007)

[Th3] _ On the structure and scarcity of alternating links and tangles, J. Knot Theory Ramifications 7(7) (1998), 981-1004. MR1654669 (99k:57031)

Department of Mathematics, Research Institute for Mathematical Sciences, Kyoto UNIVERSITY, KYOTO 606-8502, JAPAN

E-mail address: stoimeno@kurims.kyoto-u.ac.jp 